\title{
Yersinia Infection
}

\author{
Sirwan Salman Sleman* \\ Department of Microbiology, College of Veterinary Medicine, University of Sulaimani, Sulaymaniyah, Iraq
}

Received: July 15, 2015; Accepted: July 20, 2015; Published: August 15, 2015

*Corresponding author: Sirwan Salman Sleman, Department of Microbiology, College of Veterinary Medicine, University of Sulaimani, Sulaymaniyah, Iraq. Email: sirwan.sleman@univsul.edu.iq

\section{Editorial}

Microscopically, Yersinia enterocolitica is a facultative anaerobic gram negative bipolar-staining Coccobacilli from a member of the family Enterobacteriaceae. It can ferment glucose and sucrose, grow on MacConkey agar and are bile tolerant [1]. It is one of the most important human enteropathogens that have multiple $\mathrm{O}$ and $\mathrm{H}$ antigens. It has more than 50 diverse serogroups but only some of them are described to be human pathogens which are included; 0:3, 0:5, 27, 0:8, and 0:9 [2,3].

The infectious organism is recently found to become worldwide with the highest incidence in Scandinavian, other European countries [4,5] Japan and Canada [6] but it is lower in the US and the UK. The incidence of the disease decreases with the increase in age, in other words, it is commonly affected in younger children [7].

The organism has a wide range of reservoirs and is transmitted by several different routes. It is found in aquatic environments (water and marine animals such as fish, shrimps, crabs and oysters), in soil, birds, many wild and domestic animals such as pigs (most commonly), cattle, sheep, horses, deer, raccoons, rodents, rabbits, dog and cats [7].

The organism is found to transmit by many ways: one of the most important ways is that food borne transmissions, in other words, human may acquire the infection by ingestion of contaminated foods such as raw or undercooked pork and beef, eggs, cheese, unpasteurized milk, raw seafood and other infected animal products; also water born infection is reported in some countries by drinking of contaminated or untreated water because the organism is naturally found in water: person to person transmission is rarely found to occur through contact with contaminated food handlers and infected carriers or with nosocomial infections in hospitalized patients; direct or indirect contact with infected animals or their faeces may play a role in the transmission of the disease. This is common in those who are dealing with animal or working on farms such as farmers and veterinarian; exposure of skin wounds to soil containing the organism may also lead to transmission of the infection; some rare cases of blood transfusion from carriers have been reported $[6,7]$.

Yersinia enterocolitica was originally thought to be a non-human pathogen and until late $60 \mathrm{~s}$ it is found to cause gastroenteritis. The disease generally has two forms of clinical importance, which are intestinal and extra-intestinal infections. The intestinal infections involve enterocolitis, which is associated with diarrhoea (with or without blood), vomiting, fever and abdominal pain in infants and young children; acute terminal ileitis; mesenteric lymphadenitis and painful appendicitis in older children and young adults [5,7] as well as bacteremia with systemic symptoms are also seen in immunosuppressive [6] and haemochromatosis patients [8].

The organism is also rarely cause extra-intestinal infections that means at other sites of the body other than intestine, particularly as a post infectious complications including: the urinary tract infections like urethritis and cholecystitis; respiratory tract infections such as pleurisy, empyema and pneumonia; wound infections; bone and joint, such as reactive arthritis, axillary abscesses, vasculitis, endocarditis, infected mycotic aneurysm, erythema nodosum along the trunk and leg [6], pharyngitis, conjunctivitis, meningitis, osteomyelitis, pyomyositis, spleen, and liver infections $[5,6]$.

Other human pathogenic Yersinia species are Yersinia pestis and Yersinia pseudotuberculosis. Yersinia pestis is a common cause of the plague that is an acute zoonotic infectious disease which affects humans and animals. Rodents are the most common reservoir of the bacterium, rabbits are also found as a less common source and other occasional reservoirs are dogs, cats and fleas [9].

The clinical manifestations involve plagues including bubonic plague, pneumonic plague, septicemic plague, plague meningitis, and plague pharyngitis. The bubonic plague is highly contagious, which is caused by infected flea bites and it is characterized by the presence of black hemorrhagic lymph nodes in the neck and thigh region called 'Buboes'. Pneumonia, internal organ hemorrhage, gangrenes in the extremities called 'Black Death', Bacteremia, shock and death occurs. While the pneumonic plague is also highly contagious and most infectious form of the disease which is caused by inhalation of infected droplets. It is characterized by severe pneumonia associated with bloody sputum and high mortality rate usually $100 \%$. Both Septicaemic plague, disseminated intravascular coagulation and plague meningitis occurs after the bacterium invaded the bloodstream $[10,11]$. 
The disease may transmit from infected animals to human and other animals by some methods: bites from infected rodent fleas; direct contact with infected tissue or bodily fluids; through inhalation of droplets containing organism: only person to person transmissions have reported from the pneumonic plague $[12,13]$.

There are some possible control methods that may help in reducing the infectious plague: avoiding flea bites or contact by using of repellent or insecticide: controlling of rodent by removing their habit around the home and organizations; preventing pets in contact with rodent or infected fleas [13]; wearing of gloves when handling the laboratory rat and mice [14]; the infected persons should be isolated to prevent contact with other and precaution should be undertaken for those who come in close contact with the patients and they need to be monitored until the disease is controlled with antibiotics; currently no vaccines are commercially available [15].

\section{Acknowledgement}

Special thanks to the staff of Department of Microbiology and Virology, University of Manchester, UK.

\section{References}

1. Tauxe, RV. Microbiology and pathogenesis of Yersinia infections. [Cited 2011 May 24]. Available from: http://www.uptodate.com

2. Weagant SD, Feng P. Yersinia enterocolitica. U.S Food and Drug Administration. 2007. [Cited 2011 Oct 28]. Available from: http:// www.fda.gov/food

3. Nocker A, Falcao DP, Falcao JP. Yersinia. 2009. [Cited 2010 August 17]. Available from: http://waterbornepathogens.susana.org/ menubacteria/yersinia?format=pdf_

4. Bottone EJ. Yersinia enterocolitica: the charisma continues. Clin Microbiol Rev. 1997;10(2):257-76.

5. Champoux JJ, Neidhardt FC, Drew WL, Plorde JJ. Sherris Medical Microbiology: An Introduction to Infectious Diseases. 4th ed. Kenneth
J. Ryan, C. George Ray, editors. New York: The McGraw-Hill Companies; 2004.

6. Gould LH. Infectious Diseases Related to Travel: Yersiniosis. In: Brunette GW, editor.Yellow Book: CDC Health Information for International Travel 2016. New York: Oxford University Press; 2015.

7. Schoenstadt, MD. Yersinia Enterocolitica. 2009. [Cited 2009 February 10]. Available from: http://diarrhea.emedtv.com

8. Berman, J. J. Taxonomic Guide to Infectious Diseases: Understanding the Biologic Classes of Pathogenic Organisms. 1st ed. Academic Press; 2012.

9. Cleri DJ, Vernaleo JR, Lombardi LJ, Rabbat MS, Mathew A, Marton R, et al. Plague pneumonia disease caused by Yersinia pestis. Semin Respir Infect. 1997;12(1):12-23.

10.Chamberlain, NR. Infectious Diseases: Hematopoietic/ Lymphoreticular Infections: 2014. [Cited June 22, 2015]. Available from: http://www.atsu.edu/faculty/chamberlain/website/lectures/ syllabi3.htm

11. Mead PS. Infectious Diseases Related to Travel. In: Brunette GW, editor. CDC Health Information for International Travel 2016. New York: Oxford University Press; 2015.

12. Inglesby TV, Dennis DT, Henderson DA, Bartlett JG, Ascher MS, Eitzen E, et al. Plague as a Biological Weapon: Medical and Public Health Management. Working Group on Civilian Biodefense. JAMA. 2000;283(17):2281-90.

13. Schoenstadt MD. Plague Transmission. 2006. [Cited 2006 October 13]. Available from: http://plague.emedtv.com/plague/plaguetransmission.html

14. Centers for Disease Control and Prevention (CDC). Plague Prevention. 2012. [Cited June 13, 2012] Available from: http://www.cdc.gov/ plague/prevention/index.html

15. National Institute of Allergy and Infectious Diseases (NIAID). Plague. [Cited 2015 May 20]. Available from: http://www.niaid.nih.gov/ topics/plague/Pages/transmission.aspx. 